\title{
Human Airway Monohydroxyeicosatetraenoic Acid Generation and Mucus Release
}

\author{
Zvi Marom, James H. Shelhamer, Frank Sun, and Michael Kaliner, Allergic \\ Diseases Section, Laboratory of Clinical Investigation, National Institute of \\ Allergy and Infectious Diseases, National Institutes of Health, Bethesda, \\ Maryland 20205; Upjohn Company, Kalamazoo, Michigan 49007
}

\begin{abstract}
A B S TRACT The effects of 5-, 8-, 9-, 11-, 12-, and 15-monohydroxyeicosatetraenoic acid (HETE) (0.1$100 \mathrm{nM}$ ) on mucous glycoprotein release from cultured human airways were determined. Each of the HETE was an active secretagogue of mucus at concentrations >1-10 nM with 12- and 15-HETE, the most active. Both 5- and 9-hydroperoxyeicosatetraenoic acid (HPETE) were also active as secretagogues at $100 \mathrm{nM}$, although of somewhat lower potency. As cultured airways were capable of responding to HETE with mucous glycoprotein release, it was of interest to identify and quantitate airway HETE formation. Accordingly, airways were incubated with tracer quantities of $\left[{ }^{14} \mathrm{C}\right]$ arachidonate for $16-48 \mathrm{~h}$, and the spontaneous formation of 5-, 12- and 11- and/or 15-HETE was measured by high-pressure liquid chromatography. Indeed, sizeable quantities of 11- and/or 15->5$>$ 12-HETE were generated. This HETE generation was increased by the addition of $25 \mu \mathrm{g} / \mathrm{ml}$ of arachidonate and was reduced somewhat after $18-21 \mathrm{~d}$ in continuous tissue culture. Reversed anaphylaxis of human airways using anti-human $\operatorname{IgE}$ markedly increased the HETE formation, resulting in the production of micromolar concentrations of 5- and 11- and/ or 15-HETE. Thus, human airways not only are capable of responding to the presence of HETE with mucous glycoprotein release, but also generate (both spontaneously and in response to anaphylaxis) at least three species of HETE, and do so in quantities capable of acting as mucus secretagogues.
\end{abstract}

\section{INTRODUCTION}

Allergic asthma is thought to develop from immunologic stimulation of mast cells, the release and/or gen-

Address reprint requests to Dr. Zvi Marom, National Institutes of Health, Bethesda, MD 20205.

Received for publication 9 August 1982 and in revised form 21 March 1983. eration of the mediators of allergy, and the elicitation by these mediators of the pathologic responses causing airflow obstruction $(1,2)$. Of the pulmonary responses incurred by allergic provocation that contribute to asthma, mucus production appears to play an important role in determining both morbidity and mortality. To examine the mechanisms possibly contributing to mucus production from human airways, a model consisting of cultured human bronchi (3) secreting biosynthetically labeled (4) mucous glycoproteins has been developed (5). Recent studies with this model have revealed that mast cell degranulation and mediator production lead to increased mucous glycoprotein release (5). Of the mast cell-derived mediators, histamine (through an $\mathrm{H}-2$ receptor) (5), several prostaglandins (6), a mixture of synthetic monohydroxyeicosatetraenoic acids (HETE) ${ }^{1}$ (6), pure 12-HETE (6), prostaglandin-generating factor of anaphylaxis (PGF-A) (7), and the slow-reacting substances of anaphylaxis (SRS-A), leukotriene $\mathrm{C}_{4}$ and $\mathrm{D}_{4}\left(\mathrm{LTC}_{4}\right.$ and $\left.\mathrm{LTD}_{4}\right)(8)$, are all secretagogues of mucus. Whereas the precise relative contribution of each of these mediator-secretagogues to allergen-induced mucus production is unknown, the extraordinary responsivity of mucus secretion in vitro to the lipoxygenase products of arachidonate (the HETE and leukotrienes) suggests that these agents may be active in vivo as well.

Stimulation of rat mast cells leads to the generation of prostaglandin (PG) $\mathrm{D}_{2}$ and 12-HETE (9), while purified human lung mast cells may also generate SRSA (10). Anaphylaxis of human lung tissue generates $\mathrm{PGF}_{2 \alpha}, \mathrm{PGE}_{2}, \mathrm{PGI}_{2}, \mathrm{PGD}_{2}$, and thromboxane $\mathrm{A}_{2}$ (11-

${ }^{1}$ Abbreviations used in this paper: GC, gas chromatography; HETE, monohydroxyeicosatetraenoic acid; HPETE, hydroperoxyeicosatetraenoic acid; HPLC, high-pressure liquid chromatography; $\mathrm{LTC}_{4}$, leukotriene $\mathrm{C}_{4} ; \mathrm{LTD}_{4}$, leukotriene $\mathrm{D}_{4} ; \mathrm{m} / \mathrm{e}$, mass/energy ratio; $\mathrm{PG}$, prostaglandin; PGF$A$, prostaglandin-generating factor of anaphylaxis; SRS-A, slow-reacting substances of anaphylaxis. 
13), and, as yet, little is known of the specific lipoxygenase products. Both human and guinea pig lung are capable of converting arachidonic acid to HETE, human lung generating 15-HETE (14) and guinea pig lung 12-HETE (15). Certainly, human lung must also have the capability of generating 5-HETE, as activation of a 5-lipoxygenase is the initial step in SRS-A formation (16). However, the quantities and specific identification of lipoxygenase products of human airways generated during anaphylaxis are unknown.

The purpose of this paper is to describe the specific HETE generated by human airways during anaphylaxis and to demonstrate the capability of these HETE to act as secretagogues of mucus.

\section{METHODS}

$\left[{ }^{14} \mathrm{C}\right]$ Arachidonic acid $(200 \mathrm{mCi} / \mathrm{mmol})$ was obtained from New England Nuclear, Boston, MA. All other reagents and their sources have previously been described $(5,6)$. 5-, 8-, 9-, 11-, and 12-HETE and 5- and 9-hydroperoxyeicosatetraenoic acid (HPETE) were kindly provided by Dr. F. Sun (Upjohn Co., Kalamazoo, MI). 15-HETE was prepared by Dr. R. W. Bryant, and Dr. J. Vanderhook, of George Washington University School of Medicine (Wash., DC).

Preparation of human airways for culture. Human lungs were obtained at surgery primarily from tumor resection. Normal-appearing airways $2-10 \mathrm{~mm}$ in diameter were fragmented into $3 \times 5$ - $\mathrm{mm}$ replicates and cultured as described $(5,6)$. The airway explants were maintained in CMRL-1066 medium with penicillin $(158 \mu \mathrm{g} / \mathrm{ml})$, streptomycin $(250 \mu \mathrm{g} /$ $\mathrm{ml})$, and aureomycin $(25 \mu \mathrm{g} / \mathrm{ml})$ in a controlled atmosphere chamber gassed with $45 \% \mathrm{O}_{2}, 50 \% \mathrm{~N}_{2}$, and $5 \% \mathrm{CO}_{2}$, and incubated at $37^{\circ} \mathrm{C}(3)$.

Radiolabeling of mucous glycoproteins. Mucous glycoproteins were radiolabeled by incorporating $\left[{ }^{3} \mathrm{H}\right]$ glucosamine $(1 \mu \mathrm{Ci} / \mathrm{ml})$ into the culture medium. Explants were initially incubated for $16 \mathrm{~h}$ in the absence of $\left[{ }^{3} \mathrm{H}\right]$ glucosamine, washed twice with media, and then incubated with $\left[{ }^{3} \mathrm{H}\right]$ glucosamine for a 16- to 20 -h base-line period (period I). The period I supernatants were harvested, fresh culture media without $\left[{ }^{3} \mathrm{H}\right]$ glucosamine was added for an additional 4 -h period (period II), and these supernatants were subsequently harvested. Glucosamine-labeled mucous glycoproteins were precipitated from the samples by adding an equal volume of $95 \%$ ethanol. The precipitates were filtered through metricel membrane filters (6), and the retained radioactivity was determined. Analysis by gel filtration of airway supernatants before or after precipitation indicates that $>90 \%$ of all macromolecular radiolabeled glycoproteins were precipitated and filtered by this procedure.

Effects of HETE on mucous glycoprotein release. The effect of HETE on the release of $\left[{ }^{3} \mathrm{H}\right]$ glucosamine-labeled mucous glycoproteins was determined by adding these agents to cultures at the beginning of period II. A ratio of radiolabeled mucous glycoproteins released in period II/period I was determined for each sample and termed the secretory index. The effects of pharmacologic agents were determined by comparing the secretory indices of manipulated samples to matched unmanipulated control samples. HETE were maintained in absolute methanol at $-70^{\circ} \mathrm{C}$ and prepared immediately before use by evaporation to dryness under a stream of nitrogen and resuspension in culture media.
Generation of HETE by cultured human airways. Airways to be used for the generation of HETE were cultured with $\left[{ }^{14} \mathrm{C}\right]$ arachidonic acid $(0.05-1.0 \mu \mathrm{Ci} /$ plate) and/or unlabeled arachidonic acid (Nu Chek Prep, Elysian, MN) (10$25 \mu \mathrm{g} / \mathrm{plate}$ ) for 4-48 h. The culture supernatants were immediately frozen and maintained at $-70^{\circ} \mathrm{C}$ in pyrex glass tubes sealed under nitrogen. The hydroxy fatty acids were extracted from the pooled supernatants by reverse-phase chromatography on silicic acid columns (17). ODS Silica columns $(10 \times 10 \mathrm{~mm}$, SepPaks, Waters Associates, Millipore Corp., Milford, MA) were washed sequentially with methanol and water before the samples were applied. The supernatants were mixed with appropriate amounts of deuterated 5-HETE, 11-HETE, and 12-HETE standards to give a final concentration of $50 \mathrm{ng} / \mathrm{ml}$ each. The $\mathrm{pH}$ was adjusted to 4.0 with $1 \mathrm{M} \mathrm{H}_{3} \mathrm{PO}_{4}$, and the medium was passed through the SepPak column. The column was washed with $5 \mathrm{ml}$ of hexane and eluted with $10 \mathrm{ml}$ of diethyl ether. The ether extract was evaporated to dryness with a stream of nitrogen. Recovery of HETE exceeded $90 \%$.

High-pressure liquid chromatography (HPLC). The residue was dissolved in $0.05 \mathrm{ml}$ of methanol and purified by reversed-phase HPLC. HPLC was performed on a Varian Model 5000 liquid chromatograph (Varian Associates, Inc., Palo Alto, CA) linked to a Varichrom detector (Varian Associates, Inc.) operated at a wavelength of $235 \mathrm{~nm}$. The mobile phase was delivered at a flow rate of $1 \mathrm{ml} / \mathrm{min}^{-1}$. The column was washed isocratically with methanol/water/ acetic acid (80:20:0.1) for 5 min, which was followed with a linear gradient to straight methanol developed over $30 \mathrm{~min}$ (18). The HETE fraction was collected and evaporated to dryness with a stream of nitrogen.

The dried residue was dissolved in $1 \mathrm{ml}$ of diethyl ether/ methanol (10:1) and esterified with diazomethane. The methyl ester was converted to trimethylsilyl ether derivative and analyzed by a gas chromatography-mass spectrometry. Samples in which $\left[{ }^{14} \mathrm{C}\right]$ arachidonic acid were added were analyzed after gas chromatography by monitoring radioactivity.

Gas chromatography (GC) and mass spectrometry. GC was carried out with a Varian 2700 (Varian Associates, Inc.) coupled to a Packard model 594 proportional counter (Packard Instrument Co., Inc., United Technologies, Downers Grove, IL). Mass spectrometry was carried out on a Hewlett Packard 5992 GC-MS (Hewlett-Packard Co., Palo Alto, CA) operated in the selected ion monitoring mode. The GC column was $1 \%$ SE-30 ultraphase on Chromasorb W(HP) maintained at $210^{\circ} \mathrm{C}$. The signal of the masses at mass/energy ratio (m/e) 229 vs. 225 for 11 - and/or 15-HETE, m/e 301 vs. 295 for 12 -HETE, and m/e 313 vs. 305 for 5 -HETE were recorded. Quantitative estimates were computed by reference to calibration curves derived from appropriate standards. This procedure does not distinguish between 11- and 15-HETE and, therefore, the data will be presented for either or both products.

Octadeuterated $\left(D_{8}\right)$ HETE and unlabeled HETE standards were synthesized from $5,6,8,9,11,12,14,15-D_{8}$-arachidonic acid by photochemical oxygenation. They were separated and purified by preparative HPLC with a $\mu$ Porasil column (Waters Associates). The synthetic method used was essentially the same as that described previously $(19,20)$. (We are grateful to Drs. G. L. Bundy, W. P. Schneider, R. C. Kelly, and N. A. Nelson of the Department of Experimental Chemistry at The Upjohn Co. for their generous support.)

Comparison of supernatant vs. tissue HETE concentration. To estimate the relative proportion of HETE that were 
generated and remained associated with the airways in comparison with that released into the supernatant, airways were incubated for $4 \mathrm{~h}$ either in media alone or media containing $10 \mu \mathrm{g} / \mathrm{ml}$ of arachidonic acid. After completing the incubation, the tissue was resuspended in fresh medium, chilled to $4^{\circ} \mathrm{C}$, and homogenized with a polytron kinematica (Brinkman Instruments, Inc., Westbury, NY) at maximum speed for $90 \mathrm{~s}$. The homogenate was centrifuged $(2,500 \mathrm{~g}, 15 \mathrm{~min}$, $4^{\circ} \mathrm{C}$ ) and the supernatant was decanted, frozen at $-150^{\circ} \mathrm{C}$ in dry ice/acetone, and maintained at $-70^{\circ} \mathrm{C}$ under nitrogen until assayed.

Anaphylaxis of human airways. Airways anaphylaxis was produced with rabbit anti-human IgE. Antiserum directed at an IgE myeloma (PS) was partially purified by ammonium sulfate fractionation and protein A-Sepharose affinity chromatography. This material was absorbed on an IgG immunosorbent column to remove anti-light chain activity, and it formed a precipitin band against IgE heavy chain; precipitin bands against light chains or other immunoglobulins by Ouchterlony analysis were not present. A 1:100 dilution of this purified antiserum produced $20-40 \%$ histamine release in vitro from both peripheral human lung or airways. Anaphylaxis of airways with the preparation was initiated at the onset of period II, used 1:100-1:400 dilutions, and involved 4-h incubations.

Histamine was analyzed by the automated fluorometric assay (21). The identity of the fluorescent material as histamine was confirmed by diamine oxidase digestions (22).

Statistical analysis. The results for mucus release are expressed as percent change from control and represent the comparison of the secretory indices (period II/period I) of experimentally manipulated samples with matched controls. Data are presented as mean \pm SEM. The $n$ indicates the number of separate experiments combined to generate the results. In each experiment, 5-10 culture plates were used to generate each experimental point. The data were analyzed by paired sample $t$ tests with a $P$ value of $<0.05$ considered statistically significant.

\section{RESULTS}

Effects of HETEs on mucus release. 5-, 8-, 9-, 11-, 12-, and 15-HETE (0.1-100 $\mathrm{nM})$ were assessed for their effects on mucus release (Fig. 1); all of these compounds increased mucus release in a dose-dependent fashion as compared with control. Nine separate lung cultures have been exposed to these compounds, and increased mucus release has been produced in each instance with 1-100-nM concentrations. All the HETE tested were equipotent secretagogues at concentrations of 1 and $10 \mathrm{nM}$, whereas 12- and 15-HETE were the most effective stimulants at $100 \mathrm{nM}$ (12-HETE $=+73 \pm 6.7 \% ; n=4$ ).

In two separate experiments, the effects of 5- and 9-HPETE on mucus release were studied. The only

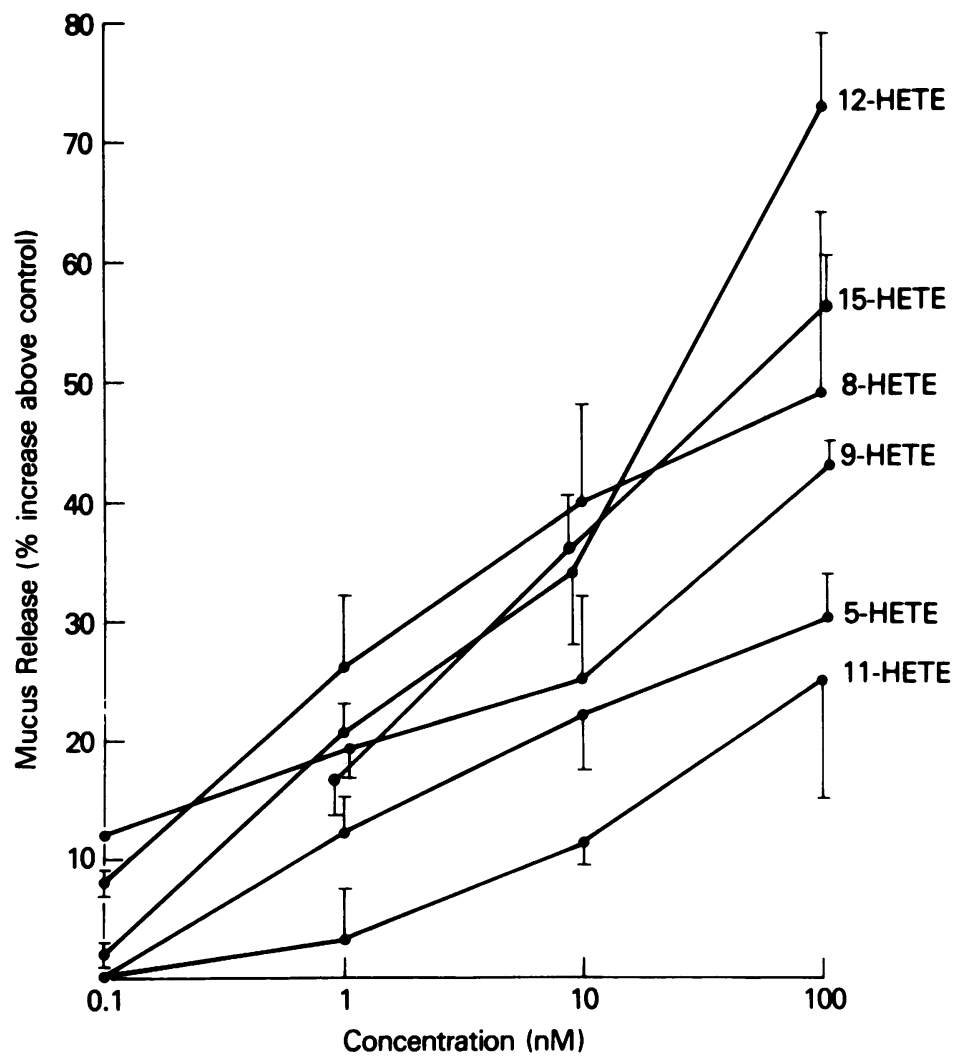

Figure 1 Dose-response of 5-, 8-, 9-, 11-, 12-, and 15-HETE on airway mucous glycoprotein release. The results are the mean \pm SEM of up to nine separate experiments. 
significant increase in mucus release was observed at concentrations of $100 \mathrm{nM}: 20 \pm 2.6 \%$ increase above control $(P<0.05)$ for 5-HPETE and 28 $P$ 1.9\% ( $P$ $<0.01)$ for 9-HPETE. Thus, these two HPETE preparations were somewhat less active than their corresponding hydroxy compounds.

Generation of HETE by human airways in vitro. The data indicated that cultured human airways secrete mucus in response to exogenously added HETE and HPETE. It was therefore of interest to examine the capacity of these same airway preparations to generate HETE. Incubation of airways with $\left[{ }^{14} \mathrm{C}\right]$ arachidonic acid for $16 \mathrm{~h}$, followed by silicic acid chromatography and GC, revealed the presence in the supernatant of two major peaks of radioactivity: the earlier peak cochromatographed with arachidonic acid while the second peak fractionated with HETE standards. Deuterated specific HETE standards were added to the incubation media as carriers, and the samples were purified by SepPak and HPLC. Mass fragmentography (selective ion monitoring) clearly showed prominent signals at $\mathrm{m} / \mathrm{e} 225,295$, and 305 , which occurred at the identical retention times as the deuterated standards at $\mathrm{m} / \mathrm{e} \mathrm{229}, 301$, and 313 . Therefore, it is likely that 11-HETE and/or 15-HETE, 12HETE, and 5-HETE were present in the incubation medium.

Analysis of tissue culture media alone or tissue culture media to which arachidonic acid was added and incubated for $16 \mathrm{~h}$ failed to reveal the presence of any detectable HETE. However, airways incubated for 4 $h$ in the presence of $25 \mu \mathrm{g} / \mathrm{ml}$ of arachidonic acid generated $32 \mathrm{ng} / \mathrm{sample}$ of 11- and/or 15-HETE and $10 \mathrm{ng}$ each of 5- and 12-HETE. Thus, airways make at least three separate HETE. In a separate experiment, airways cultured for a longer period $(48 \mathrm{~h})$ in the presence of small amounts of $\left[{ }^{14} \mathrm{C}\right]$ arachidonic acid $(0.5 \mu \mathrm{Ci})$ generated $100 \mathrm{ng} / \mathrm{sample}$ of 5 -HETE, 500 ng of 12-HETE, and 1,400 ng of 11- and/or 15-HETE.

Supernatants from airway samples that were cultured 1-3 d generated considerably more HETE than did the samples cultured 2 wk. For example, on the second day of culture, one airway preparation stimulated with $25 \mu \mathrm{g} / \mathrm{ml}$ of arachidonic acid generated $1,200 \mathrm{ng}$ of $11-$ and/or 15-HETE/sample, $500 \mathrm{ng}$ of 12-HETE, and $120 \mathrm{ng}$ of 5-HETE, while after $18 \mathrm{~d}$ in culture, the same airways under the same conditions generated only $90 \mathrm{ng}$ of 11-and/or 15-HETE, $90 \mathrm{ng}$ of 12-HETE, and $85 \mathrm{ng}$ of 5-HETE. Thus, the quantity and relative ratio of HETE generated varied with the time in culture. By $21 \mathrm{~d}$, only 5-HETE was still being produced in sizable quantities $(8 \mathrm{ng} / \mathrm{sample}$ ).

To compare the relative quantities of HETE released into the supernatant vs. those associated with the tissue, tissue incubated in buffer alone or buffer plus $25 \mu \mathrm{g} / \mathrm{ml}$ of arachidonic acid was homogenized, extracted, and assayed for HETE. The results of these homogenates were compared with the supernatants from the same samples handled in parallel. The lung tissue homogenates generated $<10 \mathrm{ng}$ each of 11 - and/ or 15-HETE, 5-HETE, and 12-HETE, while the supernatant contained $>100 \mathrm{ng}$ of each of the three HETE. Thus, the HETE generated during short-term culture are released into the supernatant.

Effect of airway anaphylaxis upon mucus release and HETE formation. In separate reports, both reversed anaphylaxis of airways with rabbit anti-IgE (anti-IgE) and the addition of exogenous arachidonic acid have been shown to stimulate increased mucus release $(5,6)$. It was of interest to examine the effects of the simultaneous administration of both stimuli upon mucus release (Table I). Anti-IgE provoked histamine release and a dose-related increase in mucus release, while arachidonic acid also acted as a secretagogue of mucus. The combination of agents induced somewhat more mucus release than either maneuver alone and did so without an accompanying increase in histamine release.

The effects of reversed anaphylaxis upon airway HETE generation was examined in three separate experiments involving three individual lung preparations (Table II). Media containing $\left[{ }^{14} \mathrm{C}\right]$ arachidonic acid cultured for $4 \mathrm{~h}$ in the absence of lung tissue had no detectable HETE while airways cultured for $4 \mathrm{~h}$ in the presence of $\left[{ }^{14} \mathrm{C}\right]$ arachidonic acid $(1 \mu \mathrm{Ci} /$ plate) produced $<5 \mathrm{ng}$ each of the three HETE. However, anaphylaxis (1:100 dilution of anti-IgE) in each instance induced sizable increases in the production of 11- and/ or 15-, 12-, and 5-HETE with the average amount generated being $163 \pm 51,88 \pm 23$, and $255 \pm 109 \mathrm{ng}$, respectively. Thus, anaphylaxis of human airways leads to HETE generation.

TABLE I

Effects of Arachidonic Acid and/or Anti-IgE Upon Mucus Release ${ }^{\circ}$

\begin{tabular}{lcc}
\hline \multicolumn{1}{c}{ Agent } & Concentration & $\begin{array}{c}\text { Mucus release } \\
\text { (Increase above control) }\end{array}$ \\
& & $\%$ \\
Arachidonic Acid & $75 \mu \mathrm{g} / \mathrm{ml}$ & $21 \pm 2.7$ \\
Anti-IgE & $1: 400$ & $26 \pm 1.9$ \\
Anti-IgE & $1: 100$ & $59 \pm 1.2$ \\
Arachidonic Acid plus & $75 \mu \mathrm{g} / \mathrm{ml}$ & \\
$\quad$ Anti-IgE & $1: 100$ & $67 \pm 4.2$ \\
\hline
\end{tabular}

- Histamine release for each sample was the following: control, $9 \pm 0.4 \mathrm{ng} / \mathrm{ml}$; arachidonic acid, $9.0 \pm 0.2 \mathrm{ng} / \mathrm{ml}$; anti-IgE (1:100), $21 \pm 4 \mathrm{ng} / \mathrm{ml}$; arachidonic acid plus anti-IgE, $24 \pm 3 \mathrm{ng} / \mathrm{ml}$. Anti-IgE (1:400) was not assayed. Each result is the mean \pm SEM of five replicate samples. 
TABLE II

Effects of Anaphylaxis on HETE Formation

by Human Airways

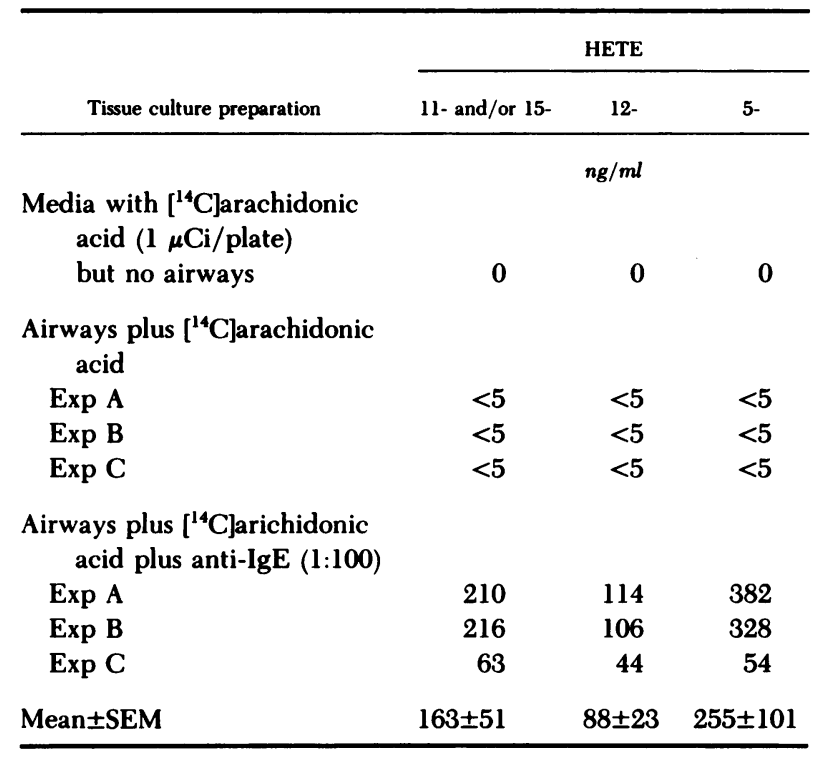

Experiments A, B, and C are three separate experiments using different lungs. All incubations were for $4 \mathrm{~h}$.

\section{DISCUSSION}

In the course of IgE-mediated allergic reactions, mast cell and basophil activation lead to the formation of an array of lipid mediators derived predominantly from arachidonic acid. The specific eicosinoids generated are determined by the tissue involved in the allergic reaction. Thus, certain cells and tissues have specific profiles of eicosinoids that they generate. For instance, mast cells themselves produce predominantly $\mathrm{PGD}_{2}$ and 12-HETE (19) while platelets produce thromboxane $A_{2}$ and 12-HETE (23). Human lung tissue is known to release $\mathrm{PGF}_{2 \alpha}$, thromboxane $\mathrm{A}_{2}, \mathrm{PGE}_{2}$, $\mathrm{PGD}_{2}, \mathrm{PGI}_{2}$, and the leukotrienes $\mathrm{LTC}_{4}, \mathrm{LTD}_{4}$, and $\mathrm{LTE}_{4}(11-13,24,25)$. Although not previously documented, it was clear that lung was also capable of producing monohydroxy oxidative products of arachidonic acid as 5-HPETE, the first stage in leukotriene formation.

Evidence is presented herein that human airways produce at least three distinct HETE: 11- and/or 15-, 5-, and 12-HETE. The synthesis of these HETE occurs spontaneously, without the need for exogenous stimulation, or may be accelerated by adding exogenous arachidonic acid or by anaphylaxis. Airways maintained in culture for $48 \mathrm{~h}$ with only trace amounts of exogenous arachidonic acid generate sizable quantities of 11- and/or 15- > 12- > 5-HETE, producing microgram quantities in the process. This synthesis may be markedly increased by adding $10-25 \mu \mathrm{g} / \mathrm{ml}$ of arachidonic acid, although the relative concentrations of the individual HETE produced remains the same: 11- and/or 15- > 12- > 5-HETE. HETE production decreased with time in culture, so that by 3 wk only trace amounts of 5-HETE were still being produced. Accordingly, all the results reported herein derive from airways cultured $<7 \mathrm{~d}$.

Although the observation that airways are capable of converting arachidonic acid to at least three species of HETE extends and confirms earlier observations involving guinea pig lung (14) and human peripheral lung (13), the capacity of anaphylaxis to stimulate HETE formation from airways is a new and potentially important observation. In three separate lung cultures undergoing anaphylaxis, 5- > 11- and/or 15- > 12HETE synthesis was appreciated. The quantities synthesized were between 88 and $250 \mathrm{ng} /$ culture, easily achieving concentrations known to be capable of stimulating mucus secretion $(6,26)$ and influencing neutrophil degranulation (27) as well as possibly modulating other airway-related events. Whereas the precise roles these HETE play in airway physiology are not known, the capacity of each of these products to stimulate mucous glycoprotein secretion suggests that they may contribute to at least this facet of allergic airways disease.

In earlier work, the ability of arachidonic acid to stimulate mucus secretion from human airways was appreciated (6). The arachidonic acid appeared to act through interaction with lipoxygenase enzymes, leading to the formation of HETE. In this earlier work, complex mixtures of synthetic oxidative products of arachidonic acid as well as biosynthetic 12-HETE were shown to be capable of stimulating mucus secretion. In this study, these observations are extended to include 5-, 9-, 11-, 12-, and 15-HETE as well as 5- and 9-HPETE. Thus, HETE at concentrations $>1-100 \mathrm{nM}$ can act as secretagogues of mucus, and these are the concentrations achieved by airways undergoing anaphylaxis.

Other eicosinoids, including a variety of PG and $\mathrm{LTC}_{4}$ and $\mathrm{LTD}_{4}$, are also secretagogues $(6,7)$. The PG act in vitro at micromolar or higher concentrations and, thus, may not be physiologically important. The leukotrienes, however, are potent secretagogues at nanomolar concentrations and, clearly, may also contribute to the mucus secretion accompanying allergic airways disease. Although the precise mechanisms controlling the initial steps in leukotriene formation are not clearly defined as yet, it appears that anaphylaxis of lung tissues is generally required to activate the specific pathways involved. HETE formation from lung tissue may therefore occur more readily than SRS-A formation, as we observed that simply culturing airway tissue or adding exogenous substrate was suf- 
ficient to lead to sizable HETE formation. Thus, it is possible that HETE production by airways occurs more commonly than does leukotriene formation and, therefore, that HETE may play a larger role in mucus production (and other lung functions as well). We conclude that airways in culture produce at least three species of HETE, that this synthesis is accelerated by anaphylaxis, and that the HETE formed act as potent mucus secretagogues.

\section{ACKNOWLEDGMENTS}

The authors gratefully acknowledge the fine typing of Ms. Joni Stefanelli, the editorial assistance of Karen Leighty, and the cooperation of the pathology and surgical departments at the Washington Hospital Center, Sibley Hospital, Suburban Hospital, Holy Cross Hospital, Walter Reed Army Medical Center, Montgomery General Hospital, and Washington Adventist Hospital in providing surgical specimens for this study.

\section{REFERENCES}

1. Kaliner, M. A., J. Blennerhasset, and K. F. Austen. 1976. Bronchial asthma. In Textbook of Immunopathology. P. A. Meischer and H. J. Müller-Eberhard, editors. Grune \& Stratton, New York. 387-401.

2. Kaliner, M. A. 1980 . Mast cell-derived mediators and bronchial asthma. In Airway Reactivity. F. E. Hargreave, editor. Astra Pharmaceuticals, Canada LTD, Mississaugo, Ontario, Canada. 175-188.

3. Barret, L. A., E. M. McDowell, A. L. Frank, C. C. Harris, and B. F. Trump. 1976. Long-term organ culture of human bronchial epithelium. Cancer Res. 36:1003-1016.

4. Boat, T. F., and J. I. Kleinerman. 1975. Human respiratory tract secretions: effect of cholinergic and adrenergic agents on in vitro release of protein and mucous glycoprotein. Chest. 67:S325-335.

5. Shelhamer, J. H., Z. Marom, and M. Kaliner. 1980. Immunologic and neuropharmacologic stimulation of $\mathrm{mu}$ cous glycoprotein release from human airways in vitro. J. Clin. Invest. 66:1400-1408.

6. Marom, Z., J. H. Shelhamer, and M. Kaliner. 1981. Effects of arachidonic acid, monohydroxyeicosatetraenoic acid, and prostaglandins on the release of mucous glycoproteins from human airways in vitro. J. Clin. Invest. 67:1695-1702.

7. Marom, Z., L. Steel, J. Shelhamer, E. Goetzl, and M. Kaliner. 1982. Prostaglandin-generating factor of anaphylaxis increases mucous glycoprotein secretion and HETE formation from human airways. Clin. Res. 30:479a. (Abstr.)

8. Marom, Z., J. H. Shelhamer, M. K. Bach, D. R. Morton, and $M$. Kaliner. 1982. Slow-reacting substances LTC and LTD $_{4}$ increase the release of mucus from human airways in vitro. Am. Rev. Respir. Dis. 126:449-451.

9. Roberts, L. J., R. A. Lewis, J. A. Oates, and K. F. Austen. 1979. Prostaglandin, thromboxane, and 12-hydroxy, 5,8,10,14-eicosatetraenoic acid production by ionophore-stimulated rat serosal mast cells. Biochim. Biophys. Acta. 575:185-192.

10. MacGlashan, D. W., Jr., R. P. Schleimer, S. P. Peters, E. S. Schulman, G. K. Adams III, H. H. Newball, and L. M. Lichtenstein. 1982. Generation of leukotrienes by purified human lung mast cells. J. Clin. Invest. 70:747751.

11. Platshon, L. F., and M. Kaliner. 1978. The effects of the immunologic release of histamine upon cyclic nucleotide levels and prostaglandin synthesis. J. Clin. Invest. 62:1113-1121.

12. Adkinson, F. N., H. H. Newball, S. Findlay, K. Adams, and L. M. Lichtenstein. 1980. Anaphylactic release of prostaglandins from human lung in vitro. Am. Rev. Respir. Dis. 121:911-920.

13. Schulman, E. S., N. F. Adkinson, and H. H. Newball. 1982. Cyclooxygenase metabolites in human lung anaphylaxis: airway versus parenchyma. J. Appl. Physiol. 53:589-595.

14. Hamberg, M., P. Hedquist, and K. Radegran. 1980. Identification of 15-hydroxy-5,8,11,13-eicosatetraenoic acid (15-HETE) as a major metabolite of arachidonic acid in human lung. Acta Physiol. Scand. 110:219-221.

15. Hamberg, M., and B. Samuelsson. 1974. Prostaglandin endoperoxides. VII. Novel transformation of arachidonic acid in guinea pig lung. Biochem. Biophys. Res. Commun. 61:942-949.

16. Parker, C. W. 1982. The chemical nature of slow-reacting substances. Adv. Inflam. Res. 4:1-24.

17. Powell, W. S. 1980. Rapid extraction of oxygenated metabolites of arachidonic acid from biological samples using octadecylsilyl silica. Prostaglandins. 20:947-957.

18. Claeys, M., N. C. Coene, A. G. Herman, G. H. Jauvenaz, and D. H. Nugteren. 1982. Characterization of lipoxygenase metabolites of arachidonic and linoleic acid in rabbit peritoneal tissue. Biochim. Biophys. Acta. 713:160169.

19. Porter, N. A., J. Logan, and V. Kontoyiannidon. 1979. Preparation and purification of arachidonic acid hydroperoxides of biological importance. J. Organic Chem. 44:3177-3180.

20. Boeynaems, J. M., A. R. Brash, J. A. Oates, and W. C. Hubbard. 1980. Preparation and assay of monohydroxyeicosatetraenoic acids. Anal. Biochem. 104:259-267.

21. Siraganian, R. P. Histamine release and assay methods for the study of human allergy. 1976. In Clinical Immunology. N. Rose and H. Friendman, editors. 1976. American Society for Microbiology, Washington, DC. 603-615.

22. Myers, G., M. Donlon, and M. Kaliner. 1981. Measurement of urinary histamine: development of methodology and normal values. J. Allergy Clin. Immunol. 67:305311 .

23. Hamberg, M., and B. Samuelsson. 1974. Novel transformation of arachidonic acid in human platelets. Proc. Natl. Acad. Sci. USA. 71:3400-3404.

24. Steel, L. K., L. F. Platshon, and M. A. Kaliner. 1979. Prostaglandin generation by human and guinea pig lung tissue: comparison of parenchymal and airway responses. J. Allergy Clin. Immunol. 64:287-293.

25. Lewis, R. A., K. F. Austen, J. M. Drazen, D. A. Clark, A. Marfat, and E. J. Corey. 1980. Slow-reacting substances of anaphylaxis: identification of leukotrienes C1 and D from human and rat sources. Proc. Natl. Acad. Sci. USA. 77:3710-3714.

26. Shelhamer, N. J., Z. Marom, F. Sun, M. K. Bach, and M. A. Kaliner. 1982. The effects of arachinoids and leukotrienes on the release of mucus from human airways. Chest. 815:S36-37.

27. Stenson, W. F., and C. W. Parker. 1980. Monohydroxyeicosatetraenoic acids (HETEs) induce degranulation of human neutrophils. J. Immunol. 124:21002104. 